\title{
A comparison study of anti food allergy of plane tree leaves extract with the chemical drug therapy in affected dogs
}

\author{
Saam TORKAN, Nima MOHAJERI, Faham KHAMESIPOUR
}

\begin{abstract}
Food allergy includes an overreaction of the immune system to certain foods or substances that trigger the immune system become confused. The purpose of this study was to evaluate the effect of oral administration of plantain leaf extract on immunity against food allergies in dogs. This study performed on 12 dogs and the dogs were divided into 3 groups. In 3 groups, 2 times a day for 5 days 10 grams of turmeric tablet was administered to food allergies occur in all categories. To first group twice daily, for 5 days, in each time $100 \mathrm{cc}$ oral plantain extract administered. In second group, prednisolone acetate used instead of plantain extract for 5 days (according to the dose of $2 \mathrm{mg} / \mathrm{kg}$ every 24 hours). And in third group (control) no medication used. Blood samples took for hematology and immunology experiments at days 0, 5 and 10 and the amount of blood was divided into two equal parts, and for hematology tests and to measure IgE
\end{abstract}

immune factors and percentage and number of phagocytosis was sent to faculty lab. The result showed that in three groups comparison, during the recovery (post- treatment), eosinophils (Eos), immunoglobulin $\mathrm{E}$ ( $\mathrm{IgE}$ ) and germ amount in Leaf extract group has no significant change in appropriation to control group. The amount of these factors in comparison with the chemical drug group (Corton) had significant change $(\mathrm{p}<0.05)$, and other factors did not change significantly during treatment $(\mathrm{p}<0.05)$. Moreover, comparing two groups of Corton and sycamore, there is significantly better changes in $\operatorname{IgE}$ and Eos factors in the plantain leaves group. Therefore, mentioned factors indicate a favorable effect of plantain leaf extract in removing the symptoms of food allergy in dogs.

Keywords: Corticosteroids, Dog, Food allergy, Immune system, Plantain leaf extract
Saam TORKAN

Department of Small Animal Internal Medicine, Faculty of Veterinary Medicine, Shahrekord Branch, Islamic Azad University, Shahrekord, Iran

Nima MOHAJERI

Faculty of Veterinary Medicine, Shahrekord Branch, Islamic Azad University, Shahrekord, Iran

Faham KHAMESIPOUR

Cellular and Molecular Research Center, Sabzevar University of Medical Sciences, Sabzevar, Iran

Young Researchers and Elite Club, Shahrekord Branch, Islamic Azad University, Shahrekord, Iran

Corresponding author:

Dr. F. Khamesipour

Phone: +989134132858

Email:Dr_Faham@yahoo.com;

Submitted/Gönderilme: 19.12.2015

Accepted/Kabul: 02.02.2016

Revised/Düzeltme: 30.01 .2016

\section{INTRODUCTION}

An allergy is a response by the body's immune system to something (called an allergen) that is not necessarily harmful in itself. Some allergic reactions are mild and harmless, but others are severe and potentially life-threatening (anaphylaxis) (1). A food allergy occurs when the body's immune system reacts abnormally to specific foods (1). Food allergy includes an overreaction of the immune system to certain foods or substances that trigger the immune system. Normally, the immune system has the task of defense against microorganisms and the production of antibodies against these antigens. Plane trees are an important source of airborne allergens in many cities of the United States and Western Europe (2). Exposure to London plane tree pollen can induce asthma, allergic rhinitis and allergic conjunctivitis (3-5); systemic reactions have occurred during immunotherapy (6). 
Plantago major (PM), also known as plantain, is a weed found in temperate zones worldwide. PM leaves have been associated with various biological properties ranging from anti-inflammatory, antimicrobial and antitumor to wound healing (7). Apart from wound healing Plantago major L. leaves have been used in the treatment of diseases related to the skin, respiratory organs, digestive organs, reproduction, the circulation, against cancer, for pain relief and against infections (7). P. major contains biologically active compounds such as polysaccharides, lipids, caffeic acid derivatives, flavonoids, iridoid glycosides and terpenoids (8). The London Plane Tree (Platanus $x$ acerifolia; 'Platanus') is a fast-growing deciduous tree, a hybrid of the Oriental Plane Tree (Platanus orientalis) and the American Sycamore (Platanus occidentalis), (9) and is commonly planted in many cities around the world.

The plane trees are widely planted to improve the microclimate (10). The plane leaves commonly known in Iran as "Barge Chenar", have been used in concentrated aromatic liquids, herbal remedies and Iranian traditional medicine to treat several disorders. They are used in Iranian folk and traditional medicines for treating some dermatological, gastrointestinal, rheumatic and inflammatory diseases (1113). Ethno-pharmacological data from eastern African traditional uses of Plantago palmata leaves suggest that some therapeutically activities could be dependent on their content in polysaccharides (14). To strengthen the immune system against food allergies many studies have been done on plantain Leaf Extract (14). The present study was undertaken to evaluate the effect of oral administration of plantain leaf extract on immunity against food allergies in dogs.

\section{MATERIAL AND METHODS}

\subsection{Material}

The study was performed on 12 dogs (age 1-5 years, Iranian native breed) that were randomly allocated to two equal treatment groups. All dogs were subjected to clinical examination and housed under uniform environment after being treated for internal parasites.

The dogs were divided into 3 groups. In 3 groups, 2 times a day for 5 days 10 grams of turmeric tablet was administered to food allergies occur in all categories. And from the level, food allergy term used. To first group twice daily, for 5 days, in each time $100 \mathrm{cc}$ oral plantain extract administered. In second group, prednisolone acetate used instead of plantain extract for 5 days (according to the dose of $2 \mathrm{mg} / \mathrm{kg}$ every
24 hours). And in third group (control) no medication used.

Blood samples were collected for both hematological and immunological examnimations at days 0, 5 and 10 and the amount of blood was divided into two equal parts and use the same container for collection of blood samples, for hematological tests, including packed cell volume (PCV), haemoglobin $(\mathrm{Hb})$, red blood cell count $(\mathrm{RBC})$, white blood cell count (WBC), counting neutrophils (Nut), lymphocytes (Lym), monocytes (Mon), eosinophils (Eos), basophils (Baso) and Band Cell and to measure IgE immune factors and percentage and number of phagocytosis was sent to faculty lab in .

\subsection{Statistics}

The results are presented as mean \pm S.E.M. and statistically analyzed by One-way ANOVA followed by the Duncan test. $P<0.05$ was considered significant.

\section{RESULTS AND DISCUSSION}

Comparison of the PCV, RBC, Hb, WBC, Nut, Lym, Mon, Eos, Band Cell, Phagocyte, IgE and Germ in three groups (plantain extract, corton and control groups) as shown in Table 1 to Table 12. The result showed that in three groups comparison, during the recovery (post- treatment), Eos, IgE and Germ amount in Leaf extract group has no significant change in appropriation to control group $(\mathrm{p}>0.05)$. The amount of these factors in comparison with the Corton group had significant change $(\mathrm{p}<0.05)$, and other factors did not change significantly during treatment $(\mathrm{p}<0.05)$.

Table 1. Comparison of the packed cell volume (PCV) in three groups

\begin{tabular}{|c|c|c|c|c|}
\hline Groups & Number & $\begin{array}{c}\text { Before } \\
\text { intervention }\end{array}$ & $\begin{array}{c}\text { Since the } \\
\text { beginning of the } \\
\text { intervention }\end{array}$ & $\begin{array}{c}\text { Recovery } \\
\text { time }\end{array}$ \\
\hline & & $\mathrm{SD} \pm$ Mean & $\mathrm{SD} \pm$ Mean & $\mathrm{SD} \pm$ Mean \\
\hline $\begin{array}{l}\text { Plantain } \\
\text { extract }\end{array}$ & 4 & $\begin{array}{c}\mathrm{ab} \\
40.5 \pm 0.70\end{array}$ & $\begin{array}{c}\mathrm{a} \\
26.5 \pm 2.12\end{array}$ & $\begin{array}{c}\mathrm{a} \\
30.5 \pm 2.12\end{array}$ \\
\hline Corton & 4 & $\begin{array}{c}\mathrm{a} \\
31.5 \pm 3.53\end{array}$ & $\begin{array}{c}\mathrm{a} \\
28.5 \pm 2.12\end{array}$ & $\begin{array}{c}\mathrm{a} \\
32 \pm 1.41\end{array}$ \\
\hline Control & 4 & $\begin{array}{c}\mathrm{b} \\
46.5 \pm 2.12\end{array}$ & $\begin{array}{c}\mathrm{a} \\
28.5 \pm 0.7\end{array}$ & $\begin{array}{c}\mathrm{a} \\
34.5 \pm 0.7\end{array}$ \\
\hline SIG & & 0.019 & 0.513 & 0.164 \\
\hline
\end{tabular}

The numbers with similar letters do not significantly different $(\mathrm{P}>0.05)$ 
Table 2. Comparison of the red blood cell count (RBC) in three groups

\begin{tabular}{|c|c|c|c|c|}
\hline Groups & Number & $\begin{array}{c}\text { Before } \\
\text { intervention }\end{array}$ & $\begin{array}{l}\text { Since the } \\
\text { beginning of the } \\
\text { intervention }\end{array}$ & $\begin{array}{c}\text { Recovery } \\
\text { time }\end{array}$ \\
\hline & & $\mathrm{SD} \pm$ Mean & $\mathrm{SD} \pm$ Mean & $\mathrm{SD} \pm$ Mean \\
\hline $\begin{array}{l}\text { Plantain } \\
\text { extract }\end{array}$ & 4 & $\begin{array}{c}a b \\
6.65 \pm 0.21\end{array}$ & $\begin{array}{c}a \\
5 \pm 0.28\end{array}$ & $\begin{array}{c}a \\
5.35 \pm 0.63\end{array}$ \\
\hline Corton & 4 & $\begin{array}{c}a \\
5.25 \pm 0.63\end{array}$ & $\begin{array}{c}\mathrm{a} \\
5.25 \pm 0.21\end{array}$ & $\begin{array}{c}\mathrm{a} \\
5.8 \pm 0.14\end{array}$ \\
\hline Control & 4 & $\begin{array}{c}\mathrm{b} \\
7.55 \pm 0.35\end{array}$ & $\begin{array}{c}\mathrm{a} \\
5 \pm 0.14\end{array}$ & $\begin{array}{c}\mathrm{a} \\
6.2 \pm 0.14\end{array}$ \\
\hline SIG & & 0.030 & 0.506 & 0.235 \\
\hline
\end{tabular}

The numbers with similar letters do not significantly different $(\mathrm{P}>0.05)$

Table 3. Comparison of the haemoglobin $(\mathrm{Hb})$ in three groups

\begin{tabular}{|c|c|c|c|c|}
\hline Groups & Number & $\begin{array}{c}\text { Before } \\
\text { intervention }\end{array}$ & $\begin{array}{l}\text { Since the } \\
\text { beginning of the } \\
\text { intervention }\end{array}$ & $\begin{array}{l}\text { Recovery } \\
\text { time }\end{array}$ \\
\hline & & $\mathrm{SD} \pm$ Mean & $\mathrm{SD} \pm$ Mean & $\mathrm{SD} \pm$ Mean \\
\hline $\begin{array}{l}\text { Plantain } \\
\text { extract }\end{array}$ & 4 & $\begin{array}{c}\mathrm{ab} \\
13.8 \pm 0.42\end{array}$ & $\begin{array}{c}a \\
10.75 \pm 0.77\end{array}$ & $\begin{array}{c}\mathrm{a} \\
11.25 \pm 0.63\end{array}$ \\
\hline Corton & 4 & $\begin{array}{c}\mathrm{a} \\
11.7 \pm 0.84\end{array}$ & $\begin{array}{c}\mathrm{a} \\
11.05 \pm 0.35\end{array}$ & $\begin{array}{c}\mathrm{a} \\
12.1 \pm 0.28\end{array}$ \\
\hline Control & 4 & $\begin{array}{c}\mathrm{b} \\
14.85 \pm 0.49\end{array}$ & $\begin{array}{c}\mathrm{a} \\
10.90 \pm 0.42\end{array}$ & $\begin{array}{c}\mathrm{a} \\
12.1 \pm 0.28\end{array}$ \\
\hline SIG & & 0.032 & 0.868 & 0.225 \\
\hline
\end{tabular}

The numbers with similar letters do not significantly different $(\mathrm{P}>0.05)$

Table 4. Comparison of the white blood cell count (WBC) in three groups

\begin{tabular}{|c|c|c|c|c|}
\hline Groups & Number & $\begin{array}{c}\text { Before } \\
\text { intervention }\end{array}$ & $\begin{array}{l}\text { Since the } \\
\text { beginning of the } \\
\text { intervention }\end{array}$ & $\begin{array}{l}\text { Recovery } \\
\text { time }\end{array}$ \\
\hline & & $\mathrm{SD} \pm$ Mean & $\mathrm{SD} \pm$ Mean & $\mathrm{SD} \pm$ Mean \\
\hline $\begin{array}{l}\text { Plantain } \\
\text { extract }\end{array}$ & 4 & $\begin{array}{c}\mathrm{a} \\
7950 \pm 494.9\end{array}$ & $\begin{array}{c}a \\
9875 \pm 601\end{array}$ & $\begin{array}{c}\mathrm{a} \\
9475 \pm 388\end{array}$ \\
\hline Corton & 4 & $\begin{array}{c}a \\
9075 \pm 530.3\end{array}$ & $\begin{array}{c}a \\
9775 \pm 1520\end{array}$ & $\begin{array}{c}a \\
9350 \pm 1272\end{array}$ \\
\hline Control & 4 & $\begin{array}{c}\mathrm{a} \\
10425 \pm 742.4\end{array}$ & $\begin{array}{c}a \\
9400 \pm 353\end{array}$ & $\begin{array}{c}\mathrm{a} \\
9250 \pm 212\end{array}$ \\
\hline SIG & & 0.058 & 0.879 & 0.959 \\
\hline
\end{tabular}

The numbers with similar letters do not significantly different $(\mathrm{P}>0.05)$
Table 5. Comparison of the neutrophils (Nut) in three groups

\begin{tabular}{|c|c|c|c|c|}
\hline Groups & Number & $\begin{array}{c}\text { Before } \\
\text { intervention }\end{array}$ & $\begin{array}{l}\text { Since the } \\
\text { beginning of the } \\
\text { intervention }\end{array}$ & $\begin{array}{c}\text { Recovery } \\
\text { time }\end{array}$ \\
\hline & & $\mathrm{SD} \pm$ Mean & $\mathrm{SD} \pm$ Mean & $\mathrm{SD} \pm$ Mean \\
\hline $\begin{array}{l}\text { Plantain } \\
\text { extract }\end{array}$ & 4 & $\begin{array}{c}\mathrm{a} \\
57.5 \pm 3.53\end{array}$ & $\begin{array}{c}\mathrm{a} \\
56.5 \pm 12.2\end{array}$ & $\begin{array}{c}\mathrm{a} \\
66.5 \pm 2.12\end{array}$ \\
\hline Corton & 4 & $\begin{array}{c}\mathrm{a} \\
71 \pm 1.41\end{array}$ & $\begin{array}{c}\mathrm{a} \\
52.5 \pm 6.36\end{array}$ & $\begin{array}{c}\mathrm{a} \\
63.5 \pm 0.7\end{array}$ \\
\hline Control & 4 & $\begin{array}{c}a \\
52 \pm 9.89\end{array}$ & $\begin{array}{c}\mathrm{a} \\
62 \pm 2.82\end{array}$ & $\begin{array}{c}\mathrm{a} \\
56.5 \pm 3.5\end{array}$ \\
\hline SIG & & 0.108 & 0.803 & 0.054 \\
\hline
\end{tabular}

The numbers with similar letters do not significantly different $(\mathrm{P}>0.05)$

Table 6. Comparison of the lymphocytes (Lym) in three groups

\begin{tabular}{|c|c|c|c|c|}
\hline Groups & Number & $\begin{array}{c}\text { Before } \\
\text { intervention }\end{array}$ & $\begin{array}{l}\text { Since the } \\
\text { beginning of the } \\
\text { intervention }\end{array}$ & $\begin{array}{l}\text { Recovery } \\
\text { time }\end{array}$ \\
\hline & & $\mathrm{SD} \pm$ Mean & $\mathrm{SD} \pm$ Mean & $\mathrm{SD} \pm$ Mean \\
\hline $\begin{array}{l}\text { Plantain } \\
\text { extract }\end{array}$ & 4 & $\begin{array}{c}\mathrm{a} \\
35 \pm 1.41\end{array}$ & $\begin{array}{c}\mathrm{a} \\
29.5 \pm 3.5\end{array}$ & $\begin{array}{c}\mathrm{a} \\
25.5 \pm 2.12\end{array}$ \\
\hline Corton & 4 & $\begin{array}{c}\mathrm{a} \\
24.5 \pm 0.70\end{array}$ & $\begin{array}{c}\mathrm{a} \\
29 \pm 1.4\end{array}$ & $\begin{array}{c}\mathrm{a} \\
28 \pm 2.8\end{array}$ \\
\hline Control & 4 & $\begin{array}{c}\mathrm{a} \\
41.5 \pm 12.02\end{array}$ & $\begin{array}{c}\mathrm{a} \\
26 \pm 2.8\end{array}$ & $\begin{array}{c}\mathrm{a} \\
26 \pm 4.24\end{array}$ \\
\hline SIG & & 0.192 & 0.477 & 0.734 \\
\hline
\end{tabular}

The numbers with similar letters do not significantly different $(\mathrm{P}>0.05)$

Table 7. Comparison of the monocytes (Mon) in three groups

\begin{tabular}{|c|c|c|c|c|}
\hline Groups & Number & $\begin{array}{c}\text { Before } \\
\text { intervention }\end{array}$ & $\begin{array}{l}\text { Since the } \\
\text { beginning of the } \\
\text { intervention }\end{array}$ & $\begin{array}{l}\text { Recovery } \\
\text { time }\end{array}$ \\
\hline & & $\mathrm{SD} \pm$ Mean & $\mathrm{SD} \pm$ Mean & $\mathrm{SD} \pm$ Mean \\
\hline $\begin{array}{l}\text { Plantain } \\
\text { extract }\end{array}$ & 4 & $\begin{array}{c}\mathrm{a} \\
3 \pm 0\end{array}$ & $\begin{array}{c}\mathrm{a} \\
1.5 \pm 0.7\end{array}$ & $\begin{array}{c}\mathrm{a} \\
3.5 \pm 0.7\end{array}$ \\
\hline Corton & 4 & $\begin{array}{c}\mathrm{a} \\
1 \pm 0\end{array}$ & $\begin{array}{c}\mathrm{a} \\
2.5 \pm 0.7\end{array}$ & $\begin{array}{c}\mathrm{a} \\
3.5 \pm 0.7\end{array}$ \\
\hline Control & 4 & $\begin{array}{c}\mathrm{ab} \\
2.5 \pm 0.7\end{array}$ & $\begin{array}{c}\mathrm{a} \\
1.5 \pm 0.7\end{array}$ & $\begin{array}{c}\mathrm{a} \\
3.5 \pm 2.12\end{array}$ \\
\hline SIG & & 0.033 & 0.385 & 1 \\
\hline
\end{tabular}

The numbers with similar letters do not significantly different $(\mathrm{P}>0.05)$ 
Table 8. Comparison of the eosinophils (Eos) in three groups

\begin{tabular}{|c|c|c|c|c|}
\hline \multirow[t]{2}{*}{ Groups } & \multirow[t]{2}{*}{ Number } & \multirow{2}{*}{$\begin{array}{c}\begin{array}{c}\text { Before } \\
\text { intervention }\end{array} \\
\mathrm{SD} \pm \text { Mean }\end{array}$} & \multirow{2}{*}{$\begin{array}{c}\begin{array}{c}\text { Since the } \\
\text { eginning of the } \\
\text { intervention }\end{array} \\
\mathrm{SD} \pm \text { Mean }\end{array}$} & \multirow{2}{*}{$\begin{array}{c}\begin{array}{c}\text { Recovery } \\
\text { time }\end{array} \\
\mathrm{SD} \pm \text { Mean }\end{array}$} \\
\hline & & & & \\
\hline \multirow{2}{*}{$\begin{array}{l}\text { Plantain } \\
\text { extract }\end{array}$} & 4 & $\mathrm{a}$ & $\mathrm{a}$ & $\mathrm{a}$ \\
\hline & & $4 \pm 1.41$ & $6.5 \pm 2.12$ & $2.5 \pm 0.7$ \\
\hline \multirow[t]{2}{*}{ Corton } & 4 & $\mathrm{a}$ & $\mathrm{a}$ & $\mathrm{a}$ \\
\hline & & $2 \pm 1.41$ & $9 \pm 4.24$ & $4 \pm 1.4$ \\
\hline \multirow[t]{2}{*}{ Control } & 4 & $\mathrm{a}$ & $\mathrm{a}$ & $\mathrm{b}$ \\
\hline & & $3.5 \pm 12.2$ & $10 \pm 5.65$ & $13.5 \pm 0.7$ \\
\hline SIG & & 0.539 & 0.726 & 0.003 \\
\hline
\end{tabular}

The numbers with similar letters do not significantly different $(\mathrm{P}>0.05)$

Table 9. Comparison of the Band Cell in three groups

\begin{tabular}{|c|c|c|c|c|}
\hline Groups & Number & $\begin{array}{c}\text { Before } \\
\text { intervention }\end{array}$ & $\begin{array}{l}\text { Since the } \\
\text { beginning of the } \\
\text { intervention }\end{array}$ & $\begin{array}{c}\text { Recovery } \\
\text { time }\end{array}$ \\
\hline & & $\mathrm{SD} \pm$ Mean & $\mathrm{SD} \pm$ Mean & $\mathrm{SD} \pm$ Mean \\
\hline $\begin{array}{l}\text { Plantain } \\
\text { extract }\end{array}$ & 4 & $\begin{array}{c}\mathrm{a} \\
0.5 \pm 0.7\end{array}$ & $\begin{array}{c}\mathrm{a} \\
1.5 \pm 0.7\end{array}$ & $\begin{array}{c}\mathrm{a} \\
1 \pm 0\end{array}$ \\
\hline Corton & 4 & $\begin{array}{c}\mathrm{a} \\
1.5 \pm 2.1\end{array}$ & $\begin{array}{c}\mathrm{a} \\
1 \pm 0\end{array}$ & $\begin{array}{c}\mathrm{a} \\
0 \pm 0\end{array}$ \\
\hline Control & 4 & $\begin{array}{c}\mathrm{a} \\
0.5 \pm 0.7\end{array}$ & $\begin{array}{c}\mathrm{a} \\
1.5 \pm 0.7\end{array}$ & $\begin{array}{c}\mathrm{a} \\
0.5 \pm 0.7\end{array}$ \\
\hline SIG & & 0.722 & 0.650 & 0.192 \\
\hline
\end{tabular}

The numbers with similar letters do not significantly different $(\mathrm{P}>0.05)$

Table 10. Comparison of the Phagocyte in three groups

\begin{tabular}{lcccc}
\hline Groups & Number & $\begin{array}{c}\text { Before } \\
\text { intervention }\end{array}$ & $\begin{array}{c}\text { Since the } \\
\text { beginning of the } \\
\text { intervention }\end{array}$ & $\begin{array}{c}\text { Recovery } \\
\text { time }\end{array}$ \\
& & SD \pm Mean & SD \pm Mean & SD \pm Mean \\
\cline { 2 - 5 } & 4 & $\mathrm{a}$ & $\mathrm{a}$ & $\mathrm{a}$ \\
Plantain \\
extract
\end{tabular}

The numbers with similar letters do not significantly different $(\mathrm{P}>0.05)$
Table 11. Comparison of the IgE in three groups

\begin{tabular}{lcccc}
\hline Groups & Number & $\begin{array}{c}\text { Before } \\
\text { intervention }\end{array}$ & $\begin{array}{c}\text { Since the } \\
\text { beginning of the } \\
\text { intervention }\end{array}$ & $\begin{array}{c}\text { Recovery } \\
\text { time }\end{array}$ \\
\cline { 2 - 4 } & & $\mathrm{SD} \pm$ Mean & $\mathrm{SD} \pm$ Mean & $\mathrm{SD} \pm$ Mean \\
\cline { 2 - 5 } $\begin{array}{lccc}\text { Plantain } \\
\text { extract }\end{array}$ & 4 & $\mathrm{a}$ & $\mathrm{a}$ & $\mathrm{a}$ \\
& & $1.9 \pm 0.2$ & $2.8 \pm 0.14$ & $2 \pm 0.2$ \\
Corton & 4 & & $\mathrm{a}$ & \\
& & $1.6 \pm 0.4$ & $2.5 \pm 0.84$ & $1.9 \pm 0.2$ \\
Control & 4 & $\mathrm{a}$ & $\mathrm{a}$ & $\mathrm{b}$ \\
& & $1.4 \pm 0$ & $1.9 \pm 0.28$ & $3.3 \pm 0.2$ \\
\hline SIG & & 0.425 & 0.347 & 0.016 \\
\hline
\end{tabular}

The numbers with similar letters do not significantly different $(\mathrm{P}>0.05)$; IgEImmunoglobulin E

Table 12. Comparison of the Germ in three groups

\begin{tabular}{lcccc}
\hline Groups & Number & $\begin{array}{c}\text { Before } \\
\text { intervention }\end{array}$ & $\begin{array}{c}\text { Since the } \\
\text { beginning of the } \\
\text { intervention }\end{array}$ & $\begin{array}{c}\text { Recovery } \\
\text { time }\end{array}$ \\
& & SD \pm Mean & SD \pm Mean & SD \pm Mean \\
\cline { 2 - 5 } $\begin{array}{lccc}\text { Plantain } \\
\text { extract }\end{array}$ & 4 & $\mathrm{a}$ & $\mathrm{a}$ & $\mathrm{a}$ \\
Corton & 4 & $10.5 \pm 4.94$ & $13 \pm 1.4$ & $17.5 \pm 2.12$ \\
& & $\mathrm{a}$ & $\mathrm{a}$ & $\mathrm{a}$ \\
Control & 4 & $\mathrm{~b}$ & $18 \pm 2.8$ & $16.5 \pm 2.12$ \\
& & $15 \pm 5.65$ & $10.5 \pm 2.1$ & $8 \pm 1.41$ \\
\hline SIG & & 0.631 & 0.089 & 0.028 \\
\hline
\end{tabular}

The numbers with similar letters do not significantly different $(\mathrm{P}>0.05)$

A study conducted on effects of Plantago major L. leaf extracts on oral epithelial cells showed that, most of the tested extracts increased the proliferation/migration of the oral epithelial cells compared to the negative control. A concentration of $1.0 \mathrm{mg} / \mathrm{mL}$ (on dry weight basis) appears to be optimal regardless of type of extract, and among the alternatives, $0.1 \mathrm{mg} / \mathrm{mL}$ was always better than $10 \mathrm{mg} / \mathrm{mL}$. Ethanol-based extracts with a concentration of $10 \mathrm{mg} / \mathrm{mL}$ had very detrimental effects on cell proliferation/migration. At the other two concentrations, ethanol-based extracts had the most beneficial effect, followed by water extracts of fresh leaves, ethanol plus water extracts of dried leaves and, finally, water extracts of dried leaves (15).

Biringanine et al. (2005) (14) reported that Immunomodulatory effects due to traces of lipopolysaccharides (LPS) in PS extracts and fractions were ruled out by the use of macrophages from $\mathrm{C} 3 \mathrm{H} / \mathrm{Hej}$ mice known to be very low responders to LPS and similar results were obtained. In addition, F2 fraction from PS50 
was particularly active in enhancing $\mathrm{NO}$ and TNF-alpha (but not IL-10) production by IFN-gamma-activated C3H/ Hej macrophages. Another study also shows that endotoxinfree methanol extracts from PM leaves, at doses of 50, 100, 250 , and $500 \mathrm{microg} / \mathrm{mL}$, were associated with $4.4+/-1,6$ $+/-1,12+/-0.4$, and $18+/-0.4$-fold increases of nitric oxide (NO) production, and increased TNF-alpha production $(621+/-31,721+/-36,727+/-36$, and $1056+/-52 \mathrm{U} / \mathrm{mL}$, respectively) by rat peritoneal macrophages, in the absence of IFN-gamma or LPS. NO and TNF-alpha production by untreated macrophages was negligible. In addition, PM extracts potentiated Con A-induced lymphoproliferation (3to 12 -fold increases) in a dose-dependent fashion, compared with the effect of Con A alone (7). These discrepancies could be explained by different detection methods with different sensitization profiles.

It has been reported that these flavonoids have potent anti-inflammatory and analgesic actions on inflammation and pain (16-18). Depending on the chemical substitutions on the flavone-skeleton, flavonoids can play a modulating, biphasic and regulatory action on inflammation and immunity. Also caffeic acid is found in the plant leaves and it has been reported that this compound has anti-inflammatory activity $(19,20)$. In the present study, comparing two groups of Corton and sycamore, the result showed significantly better changes in IgE and Eos factors in the plantain leaves group in less time than any other group can better understand the performance of essence. Mentioned factors indicate a favorable effect of plantain leaf extract in removing the symptoms of food allergy in dogs. The result is in line with a

Besin allerjisi olan köpeklerde çınar ağacı yaprak ekstresinin ile yapılan tedavinin kimyasal ilaç tedavisi ile karşılaştırılması

Öz

Besin allerjisi, bağışıllık sisteminin bazı besinler ya da besin bileşenlerinee karşı gösterdiği şiddetli reaksiyon olarak tanımlanabilir. $\mathrm{Bu}$ çalışmanın amacı, besin allerjisi olan köpeklerde sinirli ot yaprak ekstresinin oral yoldan kullanılması sonucunda elde edilecek bağışıllık sistemi cevabının değerlendirilmesidir. Bu çalışma 12 köpek üzerinde yapılmış ve köpekler 3 gruba bölünmüştür. Her üç gruba beş gün boyunca günde 2 kere 10 gramlık tabletler halinde çınar ağacı yaprak ekstresi uygulaması yapılarak deney hayvanlarında besin allerjisi oluşturulmuştur. İlk gruba; beş gün boyunca, günde 2 kere 100'er cc sinirli ot ekstresi oral yoldan uygulanmıștır (Sycamore). İkinci gruba; beş gün boyunca, 24 saatte bir 2 $\mathrm{mg} / \mathrm{kg}$ dozda prednizolon asetat uygulanmışıı (Corton). Kontrol grubu olan üçüncü gruba ise tedavi uygulanmamıştır. Köpeklerin kan örnekleri hematoloji ve immünoloji report by Samuelsen (2000) (8) showed, A range of biological activities has been found from plant extracts including wound healing activity, anti-inflammatory, analgesic, antioxidant, weak antibiotic, immunomodulating and antiulcerogenic activity. Some of these effects may attribute to the use of this plant in folk medicine.

The regulation of immune parameters induced by plant extracts may be clinically relevant in numerous diseases including food allergy. The result showed that in three groups comparison, during the recovery (posttreatment) Eos, IgE and Germ amount in has no significant change in appropriation to control group. These results suggest that IgE and Eos factors indicate a favorable effect of plantain leaf extract in removing the symptoms of food allergy in dogs. The amount of Eos, IgE and Germ factors in Corton group had significant change in comparison with the Leaf extract group during treatment. Moreover, comparing two groups of Corton and sycamore with plantain leaves group showed significantly better changes in $\operatorname{IgE}$ and Eos factors in the plantain leaves group.

\section{CONCLUSION}

In general, the pharmacological activities of the plantain leaf extract showed better effects but it is not clear whether these plant constituents are the only contributing components of this extract. Therefore, further pharmacological and biological studies are needed to determine what compounds are responsible removing the symptoms of food allergy.

\section{CONFLICT OF INTERESTS}

The authors declare that there is no conflict of interests regarding the publication of this paper.

laboratuvarında değerlendirilmek üzere çalışmanın 0., 5., ve 10. günlerinde alınmıştır. Alınan kan örneklerinin her biri iki eşit kısma ayrılarak hematolojik analizler, IgE ve diğer immünolojik faktörlerin ve fagositoz sayısı ve oranının çalışılması için fakülte laboratuvarına gönderilmiştir. Üç gruba ait verilerin incelenmesi ile elde edilen sonuçlara göre; iyileşme süreci (tedavi sonrasi), eosinofil (Eos), immunoglobulin E (IgE) ve Germ oranı kontrol grubuna göre yaprak ekstresi alan grupta değişiklik göstermemiştir. Yukarıda bahsi geçen parametrelere ait veriler kimyasal ilaç ile tedavi edilen gruba (Corton) ait sonuçlarla karşılaştırıldığında ise anlamlı bir değişiklik ( $\mathrm{p}<0.05)$ olduğu görülmüş ancak diğer faktörler değişmemiştir ( $\mathrm{p}<0.05)$. Corton ve sycamore gruplarına ait sonuçlar karşılaştırıldığında ise IgE ve Eos değerleri sinirli ot ekstresi ile tedavi edilen grupta (sycamore) olumlu yönde değişmiştir. Elde edilen sonuçlar, sinirli ot ekstresinin köpeklerde besin alerjisine ait belirtilerin baskılanması için umut vadeden bir tedavi olduğunu göstermektedir.

Anahtar kelimeler: Kortikosteroitler, köpek, besin allerjisi, bağışıklık sistemi, Sinirli ot yaprak ekstresi. 


\section{REFERENCES}

1. Roger H. Allergy - General Overview. Patient. 2015; 39: 1-6.

2. Asturias J, Ibarrola I, Eraso E, Arilla MC, Martínez A. The major Platanus acerifolia pollen allergen Pla a 1 has sequence homology to invertase inhibitors. Clin Exp Allergy 2003; 33: 978-85.

3. Bousquet J, Hejjaoui A, Becker WM, Cour P, Chanal I, Lebel B, Dhivert H, Michel FB. Clinical and immunologic reactivity of patients allergic to grass pollens and to multiple pollen species. I. Clinical and immunologic characteristics. J Allergy Clin Immunol 1991; 87: 737-46.

4. Carretero Aníbarro P, Juste Picón S, García González F, Alloza Gómez P, Pérez Giménez R, Blanco Carmona J, Reinares Ten C, Vicente Serrano J, Bascones O. Allergenic pollens and pollinosis in the city of Burgos. Alergol Inmunol Clin 2005; 20: 90-4.

5. Belver MT, Caballero MT, Contreras J, Cabañas R, Sierra E, Madero R, López Serrano MC. Associations among pollen sensitizations from different botanical species in patients living in the northern area of Madrid. J Investig Allergol Clin Immunol 2007; 17: 157-9.

6. Hejjaoui A, Ferrando R, Dhivert H, Michel FB, Bousquet J. Systemic reactions occurring during immunotherapy with standardized pollen extracts. J Allergy Clin Immunol 1992; 89: 925-33.

7. Gomez-Flores R, Calderon CL, Scheibel LW, TamezGuerra P, Rodriguez-Padilla C, Tamez-Guerra R, Weber RJ. Immunoenhancing properties of Plantago major leaf extract. Phytother Res 2000; 14: 617-22.

8. Samuelsen AB. The traditional uses, chemical constituents and biological activities of Plantago major L. A review. J Ethnopharmacol 2000; 71: 1-21.

9. Nixon KC, Poole JM. Revision of the Mexican and Guatemalan species of Platanus (Platanaceae). Lundellia 2003; 6: 103-37.

10. Pourkhabbaz A, Rastin N, Olbrich A, Langenfeld-Heyser $\mathrm{R}$, Polle A. Influence of environmental pollution on leaf properties of urban plane trees, Platanus orientalis L. Bull
Environ Contam Toxicol 2010; 85: 251-5.

11. Ebn-e Sina A. Ghanoon dar teb. Vol. 2. Tehran: Soroosh Press, pp. 119-120, 1988.

12. Zargari A. Medicinal plants. Vol. 4. Tehran: Tehran University Publications, pp. 469-470, 1990.

13. Tonekaboni SMM. Tohfatol momenin. Tehran: Nashr-e Shahr, p. 200, 2007.

14. Biringanine G, Vray B, Vercruysse V, Vanhaelen-Fastré R, Vanhaelen M, Duez P. Polysaccharides extracted from the leaves of Plantago palmata Hook.f. induce nitric oxide and tumor necrosis factor-alpha production by interferongamma-activated macrophages. Nitric Oxide 2005; 12: 1-8.

15. Zubair M, Ekholm A, Nybom H, Renvert S, Widen C, Rumpunen K. Effects of Plantago major L. leaf extracts on oral epithelial cells in a scratch assay. J Ethnopharmacol 2012; 141: 825-30.

16. Palanichamy S, Nagarajan S. Analgesic activity of Cassia alata leaf extract and kaempferol 3-O-sophoroside. J Ethnopharmacol 1990; 29: 73-8.

17. Parveen Z, Deng Y, Saeed MK, Dai R, Ahamad W, Yu YH. Anti-inflammatory and analgesic activities of Thesium chinense Turcz extracts and its major flavonoids, kaempferol and kaempferol-3-O-glucoside. Yakugaku Zasshi 2007;127:1275-9.

18. Chirumbolo $\mathrm{S}$. The role of quercetin, flavonols and flavones in modulating inflammatory cell function. Inflamm Allergy Drug Targets 2010; 9: 263-85.

19. Da Cunha FM, Duma D, Assreuy J, Buzzi FC, Niero R, Campos MM, Calixto JB. Caffeic acid derivatives: in vitro and in vivo anti-inflammatory properties. Free Readical Res 2004; 38:1241-53.

20. Norata GD, Marchesi P, Passamonti S, Pirillo A, Violi F, Catapano AL. Anti-inflammatory and anti-atherogenic effects of catechin, caffeic acid and trans-resveratrol in apolipoprotein E deficient mice. Atherosclerosis 2007; 191: 265-71. 